\title{
A Pregnant Patient with Brittle Type 1 Diabetes Successfully Managed by CSII Therapy with Insulin Aspart
}

\author{
Chigusa Higuchi' ${ }^{1}$, Atsuhito Tone ${ }^{1 *}$, Izumi Iseda' ${ }^{1}, K^{2}$ iko Tsukamoto ${ }^{1}$, Akihiro Katayama $^{2}$, Jun Wada ${ }^{2}$, Kenichi Shikata ${ }^{2}$ and Kazuyuki Hida
}

${ }^{1}$ Department of Diabetes and Metabolism, National Hospital Organization, Okayama Medical Center, Okayama, Japan

${ }^{2}$ Department of Medicine and Clinical Science, Okayama University Graduate School of Medicine, Dentistry and Pharmaceutical Sciences, Okayama, Japan

Keywords: CSII; Insulin aspart; Type 1 diabetes; Pregnancy; Gestation

\section{To the Editor}

Continuous subcutaneous insulin infusion (CSII) therapy is widely accepted for brittle type 1 diabetes, since it has the benefit of less frequency of hypoglycemia and better management of the dawn phenomenon compared to multiple daily insulin injection (MDI) therapy. On the other hand, insulin aspart, one of the rapid-acting insulin analogs, was approved for Pregnancy Category B rating by the United States Food and Drug Administration (FDA) in 2007, whereas the FDA created the rating system and approved a Pregnancy Category B rating for human regular insulin in 1979.

However, the safety and efficacy of insulin aspart used in CSII therapy has not been established in pregnant type 1 diabetic patients. Here, we report the case of a pregnant patient with brittle type 1 diabetes successfully managed by conversion from MDI therapy to CSII therapy with insulin aspart.

A 28-year-old Japanese woman was treated for a 21-year history of type 1 diabetes (Height $152 \mathrm{~cm}$, weight $48 \mathrm{~kg}$, and BMI $20.8 \mathrm{~kg} / \mathrm{m}^{2}$ ). She had no diabetic-related complications. She received insulin aspart at a dose of 5-6 units before each meal plus insulin glargine at a dose of 11 units nightly at bedtime. She had recurrent severe hypoglycemia, which often required intravenous glucose administration with disturbance of consciousness. Her pregnancy was confirmed at 8 weeks of gestation. Then laboratory studies showed HbA1c, 6.6\%; 1,5-AG, $1.6 \mu \mathrm{g} / \mathrm{ml}$ (normal range 14.0-46.0); fasting serum C-peptide, $<0.1 \mathrm{ng} / \mathrm{ml}$ (normal range 0.61-2.09); GAD antibody, 2.0unit $/ \mathrm{ml}$ (normal range $<1.5$ ); and insulin antibody, $6.0 \%$ (normal range $<7.0$ ).

She was admitted to our hospital and the therapy was switched from single daily injection of insulin glargine to NPH insulin twice a day, since safety of insulin glargine in pregnant patients has not been established. However, her glycemic control had been unimproved after the change in therapy. So the treatment was changed from MDI to CSII therapy with insulin aspart using Paradigm 712 insulin pump (Medtronic Japan, Tokyo, Japan). The frequency of hypoglycemia was markedly diminished after the initiation of CSII therapy and it occurred only twice or three times a month during pregnancy. HbA1c levels decreased to $4.8-5.2 \%$ and the level of $1,5-\mathrm{AG}$ increased to $3.3-3.8 \mu \mathrm{g} / \mathrm{ml}$ throughout gestation. The coefficient of variance (CV) of FBS successively measured for 2 weeks decreased from 30.7 before the CSII therapy to 19.3 in mid pregnancy and 19.7 in late pregnancy, suggesting that glycemic variability was ameliorated after the induction of the CSII therapy. She gave birth to a male infant weighing $3342 \mathrm{~g}$ at 40 weeks gestation without any complications. And to date, she has received CSII therapy and maintained stable glycemic control for 6 months since childbirth.

CSII is useful therapy for brittle type 1 diabetes worldwide.
There have been some reports about the efficacy of CSII therapy for pregnant diabetic patients. Lenhard and Reeves [1] reported that CSII therapy decreased the frequency of hypoglycemia and improved glycemic variability, resulting in beneficialness for pregnant women with diabetes. On the other hand, there have been several studies comparing the safety and efficacy of insulin analogs. It was reported that symptomatic episodes of hypoglycemia were significantly fewer with insulin aspart than with insulin lispro administrated by CSII [2]. Plank et al. [3] also reported that there was a trend towards a lower rate of hypoglycemia in patients with type 1 diabetes receiving insulin aspart $(8.3 \%$ ) compared with insulin lispro (20.8\%). In addition, Kurtzhals et al. [4] reported that insulin aspart showed a slightly lower IGF-1 receptor affinity than that of human insulin and that insulin lispro was 1.5 -fold more potent in biding to the IGF-1 receptor than human insulin, though the mitogenic potencies, which seemed to correlate with the IGF-1 receptor binding affinities, were similar between insulin aspart and insulin lispro. Based on these previous studies, we selected CSII therapy with insulin aspart for pregnant brittle diabetes, resulting in successful glycemic management in highrisk pregnancy.

No potential conflict of interest relevant to this article was reported.

\section{References}

1. Lenhard MJ, Reeves GD (2001) Continuous subcutaneous insulin infusion: a comprehensive review of insulin pump therapy. Arch Intern Med 22: 2293 2300.

2. Bode B, Weinstein R, Bell D, McGill J, Nadeau D, et al. (2002) Comparison of insulin aspart with buffered regular insulin and insulin lispro in continuous subcutaneous insulin infusion: a randomized study in type 1 diabetes. Diabetes Care 25: 439-444.

3. Plank J, Wutte A, Brunner G, Siebenhofer A, Semlitsch B, et al. (2002) A direct comparison of insulin aspart and insulin lispro in patients with type 1 diabetes. Diabetes Care 25: 2053-2057.

4. Kurtzhals P, Schaffer L, Sorensen A, Kristensen C, Jonassen I, et al. (2000) Correlations of receptor binding and metabolic and mitogenic potencies of insulin analogs designed for clinical use. Diabetes 49: 999-1005.

*Corresponding author: Atsuhito Tone, MD., PHD, Department of Diabetes and Metabolism, National Hospital Organization, Okayama Medical Center, 1711-1 Tamasu, Kita-ku, Okayama 701-1192, Japan, Tel: (+81) 86-294-9911; Fax: (+81) 86-294-9255;E-mail: aitone@okayama3.hosp.go.jp

Received July 24, 2010; Accepted September 06, 2010; Published September 06, 2010

Citation: Higuchi C, Tone A, Iseda I, Tsukamoto K, Katayama A, et al. (2010) A Pregnant Patient with Brittle Type 1 DiabetesSuccessfully Managed by CSI Therapy with Insulin Aspart. J Diabetes Metab 1:104. doi:10.4172/2155-6156.1000104

Copyright: $\odot 2010$ Higuchi $C$, et al. This is an open-access article distributed under the terms of the Creative Commons Attribution License, which permits unrestricted use, distribution, and reproduction in any medium, provided the original author and source are credited. 